\title{
EDITORIAL
}

\section{Medical Management of Stable Angina: Systematic Therapeutic Approach}

Stable angina is the commonest manifestation (about 60\%) of ischaemic heart disease (IHD). $30 \%$ of patients revascularized for stable CAD continue to experience angina symptoms. Several drugs are now available for the treatment of stable angina. Current ACC \& ESC guidelines suggest a step by step approach for the medical treatment of stable angina but do not suggest to guiding choice within each step. Kallistratos et al ${ }^{1}$ in a recent article suggested a systematic therapeutic approach tailored to patients' cardiovascular profiles taking into account their risk factors and comorbidities. We shall discuss this in this editorial.

First of all, we need to consider heart rate (HR) and systolic blood pressure (SBP).

If the $\mathrm{HR}$ is $>60 \mathrm{bpm}$ and SBP is $>120$ the $1^{\text {st }}$ line antianginal therapy will be beta-blocker (BB) or Non DHP calcium channel blocker (CCB) such as diltiazem. In the $2^{\text {nd }}$ step DHP or ranolazine or ivabradine can be added if needed to control angina. LA nitrates or nicorandil or trimetazidine can be used in the $3^{\text {rd }}$ step. In order to initiate non DHP CCB ejection fraction needs to be normal. Ivabradine can only be initiated if HR is $>70 \mathrm{bpm}$.

If the HR is $>60 \mathrm{bpm}$ but SBP is $<120$, ranozaline or ivabradine is the $1^{\text {st }}$ choice. Trimetazidine can be added in the $2^{\text {nd }}$ step.

If the HR is $<60 \mathrm{bpm}$ but SBP is $>120$, DHP such as amlodipine is the $1^{\text {st }}$ choice. Ranolazine can be added in the $2^{\text {nd }}$ step. LA nitrates or nicorandil or trimetazidine can be used in the $3^{\text {rd }}$ step.

If the HR is $<60 \mathrm{bpm}$ and SBP is $<120$, Ranolazine is the initial choice. Trimatazidine can be added in the $2^{\text {nd }}$ step.

Having considered HR \& SBP we need to consider specific conditions associated with stable angina in the next step in order to refine drug choices.

$33 \%$ patients with stable CAD suffer from diabetes. Ranolazine has favourable effects in reduction of $\mathrm{HbA} 1 \mathrm{c}$. Vasodilating BBs present a favourable metabolic profile since they improve insulin sensitivity $\&$ do not cause deleterious effects on lipid profile. Trimetazidine has some beneficial effect but majority of the studies had a small sample size. Ivabradine, nicorandil \& CCBs have neutral metabolic profile \& can be used safely.

$70 \%$ of heart failure with reduced ejection fraction ( $\mathrm{HFrEF}$ ) is directy lined to $\mathrm{CAD}$. In these patients $\mathrm{BB} \&$ ivabradine is the preferred choice. Nitrates, nicorandil \& DHP CCBs such as amlodipine are safe. The use of hydralazine/ isosobide dinatrate may elicit angina attack. The safety of ranolazine is uncertain $\&$ should be used with caution

In patients with stable angina and atrial fibrillation (Af) $\mathrm{BB}$ or non DHP CCBs are preferred. Ranolazine seems to suppress Af and supraventricular arrhythmias in general. Ivabradine is ineffective in Af.

Usually patients with stable angina need more than one drug to suppress angina symptoms. Not all antianginal drugs can be confined. Combining ivabradine, ranolazine \& nicorandil is not recommended due to unknown safety profile. The co-administration of ivabradine with non-DHP CCBs is contraindicated (SIGNIFY trial) ${ }^{2}$. Diltiazem \& verapamil are moderate CYP3A4 inhibitors which metabolizes ivabradine.

We hope the suggestions by Kallistratos et al published recently will improve our management of patients with stable angina in daily practice taking into account the various conditions \& comorbidities in an individual.

\section{Manzoor Mahmood $^{\mathbf{1}}$ \& Harisul Hoque $\mathbf{2}^{2}$ \\ ${ }^{1}$ Associate Professor of Cardiology, BSMMU, Dhaka, \\ ${ }^{2}$ Professor of Cardiology, BSMMU, Dhaka}

\section{References:}

1. Kallistratos MS, Poulimenos LE, Manolis AJ. Stable angina pectoris: which drugs or combinations to use in which patients. E-Journal of Cardiology Practice. 2017 June; 15(8).

2. Fox K, Ford I, Steg PG, Tardif JC, Tendera M, Ferrari R; SIGNIFY Investigators. Ivabradine in stable coronary artery disease without clinical heart failure. N Engl J Med. 2014 Sep 18; 371(12):1091-9. 